\title{
A Circularly Polarized Sector Antenna for Mobile Base Station Using Single Feed with Metallic Rod EBG
}

\author{
Piyaporn Krachodnok, Rangsan Wongsan, Nuchanart Fhafhiem* \\ School of Telecommunication Engineering, Institute of Engineering, Suranaree University of Technology, \\ Nakhon Rachasima, Thailand \\ Email: priam@sut.ac.th, rangsan@sut.ac.th, ${ }^{*}$ m5140732@g.sut.ac.th
}

Received 7 October 2014; revised 25 November 2014; accepted 5 December 2014

Copyright (C) 2014 by authors and Scientific Research Publishing Inc.

This work is licensed under the Creative Commons Attribution International License (CC BY). http://creativecommons.org/licenses/by/4.0/

(c) () D Den Access

\section{Abstract}

The propose of this paper is to design the circularly polarized antenna for mobile base station, using a curved strip dipole associated with $U$-shaped reflector and multi-layer metallic rod electromagnetic band gap (EBG). It can be used to simplify the single feed system of an antenna. The advantages of this proposed antenna are easy fabrication and installation, high gain and light weight. Moreover, it provides a sectoral radiation pattern, a main beam having a narrow beam width in the vertical direction and wider beamwidth in the horizontal direction, which are appropriate for mobile phone base station. The half-power beam in the H-plane and E-plane are 60 and 14.4 degrees, respectively. The antenna is designed and analyzed by using a computer simulation technology (CST). In addition, $S_{11}$, axial ratio, radiation pattern and gain are displayed. The designed technique could be confirmed by a measurement resulting from prototype antenna corresponding to simulation results. The proposed antenna has a bandwidth covering the frequency range of $1870-2170 \mathrm{MHz}$, and the gain of the antenna increases up to $15.11 \mathrm{dBi}$.

\section{Keywords}

Sector Antenna, Curved Strip Dipole, Electromagnetic Band Gap, Resonator Antenna, Circular Polarization

\section{Introduction}

Nowadays wireless communications have been developed for entertainment, education, economic, health and

"Corresponding author.

How to cite this paper: Krachodnok, P., Wongsan, R. and Fhafhiem, N. (2014) A Circularly Polarized Sector Antenna for Mobile Base Station Using Single Feed with Metallic Rod EBG. Open Journal of Antennas and Propagation, 2, 44-53. 
industry. Mobile technology is the most popular wireless communication and has progressed rapidly from $1 \mathrm{G}$ to $4 \mathrm{G}$. The development of antennas with new performances becomes currently imperatively essential for the new services and network of telecommunication. Therefore, the purpose of this paper is to design a high gain antenna for a mobile base station; also, the antenna requirements are as follows [1]:

- Sector antenna gain: $13-16 \mathrm{~dB}$;

- Sector antenna half power beamwidth: 60 - 65 degree or 90 degree;

- Impedance: $50 \mathrm{Ohm}$.

To achieve requirements, there is an interest in the dipole antenna in wireless communication systems because of its broadband characteristics, elementary structure and simple concept [2]-[4]. Presently, the dipole antenna extensively used in practical is S-shaped dipole antenna [5]. Moreover, the dipole is bent into a V-shape to achieve the maximum gain when it is properly aligned with the curvature of the earth [6]. Then, V-shape is compared with the upside bell dipole antenna, and it seems that the upside bell dipole can reduce the side lobe of the dipole [7] [8]. However, the dipole antenna has low gain and its radiation pattern is an omni-direction. This argues that, if we can design the antenna to increase a predefined directive gain, it will have more efficiency for field radiating.

Many new technologies have appeared in the current antenna design and one exciting discovery is the development of Electromagnetic Band Gap (EBG) structures [9]. The EBG structure applications in the antenna designs have become a thrilling topic for antenna engineering. It's a matter of the EBG structure technology, a new technology for the improvement of the antenna performances applicable for frequency spectrum of which extremely wide covered from the acoustic until the optical frequencies. Besides, EBG structures known as photonic crystals are also used to improve the performance of the antenna. The EBG can be applied to grating, frequency selective surface (FSS) and so on. Moreover, the EBG is not only used to a reflector plane [10]-[12], but also adapted for a superstrate of the primary radiator with reflector plane [13]-[16]. When EBG is used to superstrate on excitation feed with reflector plane, the advantage of EBG resonator antenna is the gain enhancement and can be increased the radiation efficiency [17] [18]. The other important feature of the primary radiator with EBG is its ability to generate linearly-polarized waves to radiate a circularly polarized (CP) wave required in many communication environments, such as the dipole antenna placed on EBG reflector plane at a $45^{\circ}$ angle [19] [20] and the microstrip antenna with dual feed in the cavity wall [21] [22]. As mentioned above, when a dipole antenna is oriented along direction on EBG reflector plane, it seems that the directive gain is not suitable for a cellular system. In other words, if we use a microstrip antenna with dual feeding, it has more complexity. Therefore, this paper presents a curved strip dipole antenna placed on the U-shaped conductor plane at a $45^{\circ}$ angle. In this proposed antenna, the directive gain is increased in $x$ and $y$ axises by using metallic rod EBG and the circular polarization is generated by using the EBG polarizer.

The rest of this paper is as follows. After brief introduction, the configuration geometry of a curved strip dipole and the proposed antenna is shown in Section 2. Then, the analysis of the resonator is presented in Section 3. Next, the simulated and measured results of the proposed antenna are discussed in Section 4. Finally, the conclusions are given in Section 5.

\section{The Circular Polarized Resonator Antenna Configuration}

The detailed geometry and parameters of resonator antenna using curved strip dipole antenna with metallic rod EBG are illustrated in Figure 1 and Table 1, respectively. First, an upturned curved strip dipole antenna was designed to resonate around $2100 \mathrm{MHz}$ and it is constructed of a copper plate (a $1 \mathrm{~mm}$ thickness). Next, it is mounted over an inexpensive curved polyvinyl chloride (PVC) with the dielectric constant of 3.4. The feed center of this antenna is connected at $\varphi=\pi / 2$. The half-wavelength of curved strip dipole antenna is $L_{d}=\pi a$. Then, U-shaped reflector plane with the size of $300 \mathrm{~mm} \times 950 \mathrm{~mm}$ is added behind the $45^{\circ}$ oriented curved strip dipole to control the radiation pattern to be directional pattern and to reduce the side and back lobe. For the gain enhancement and the polarization development, the multi-layers metallic rod EBG structure is added in this design to perform as the superstrate. The configuration of three EBG layers consist of EBG polar H (in $x$-axis), EBG polar V (in $y$-axis), and EBG polarizer. For design studied here, the lower EBG polar V layer is made up of five aluminium rods of $3.85 \times 950 \times 6 \mathrm{~mm}^{3}$ periodicity. The upper EBG polar $\mathrm{H}$ layer is composed of nineteen aluminium rods of $300 \times 13.26 \times 1 \mathrm{~mm}^{3}$ periodicity. Furthermore, the polarizer layer is constituted of five aluminium rods of $3.85 \times 950 \times 2.4 \mathrm{~mm}^{3}$ periodicity; it has been located on the top layer. When, the superstrate is 


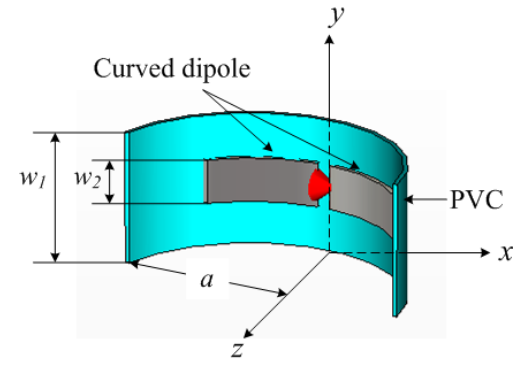

(a)



(b)

Figure 1. The geometry of (a) an upturned curved strip dipole and (b) the proposed antenna.

Table 1. Parameter of the proposed antenna.

\begin{tabular}{lc}
\hline \multicolumn{1}{c}{ Parameters } & Size (mm) \\
\hline$a:$ radius of a curved strip dipole & 34 \\
$L:$ length of a curved strip dipole & 82.81 \\
$w_{1}:$ width of a curved strip dipole & 15 \\
$w_{2}:$ width of a polyvinylchoride (PVC) & 30 \\
$A_{1}:$ width of EBG polar $\mathrm{H}$ & 13.26 \\
$g_{1}:$ the gap between the metallic rods of EBG polar H & 29.83 \\
$t_{1}:$ length of EBG polar $\mathrm{H}$ & 300 \\
$A_{2}:$ width of EBG polar V & 3.85 \\
$g_{2}:$ the gap between the metallic rods of EBG polar V and EBG polarizer & 46.42 \\
$t_{2}:$ length of EBG polar V and EBG polarizer & 950 \\
$t_{3}:$ the high of U-shaped reflector & 50 \\
$h_{1}:$ distance between EBG polar H and U-shaped reflector & 67 \\
$h_{2}:$ distance between EBG polar V and U-shaped reflector & 60 \\
$d_{1}:$ distance between EBG polar $\mathrm{H}$ and EBG polar V & 4.5 \\
$d_{2}:$ distance between EBG polar V and EBG polarizer & 30 \\
\hline
\end{tabular}

placed over a radiating element with the gap $h_{2}$ of $63 \mathrm{~mm}$, the circularly polarized resonator antenna is defined. It is possible to generate a 90 degree phase shift between the electric field in the $x$ and $y$ axis.

\section{Analysis of the Resonator Antenna}

This section shows the resonator antenna, which consists of three components. There are a superstrate, a reflector plane, and a curved strip dipole antenna for the excitation source. The superstrate forms a 1-D EBG structure where periodic conducting pattern are metallic rods. It behaves as the EBG array at the antenna operating frequencies. A curved strip dipole antenna is placed between PEC reflector plane and 1-D EBG as shown in Figure 2. The radiation of feeding antenna has a center point at $P$, whereas the radiation pattern of a curved strip dipole antenna is $f(\theta)$. In addition, the distance between the PEC reflector plane and EBG is $\mathrm{h}$, and reflection coefficient of EBG is $\gamma e^{j \phi_{\mathrm{EBG}}}$. 


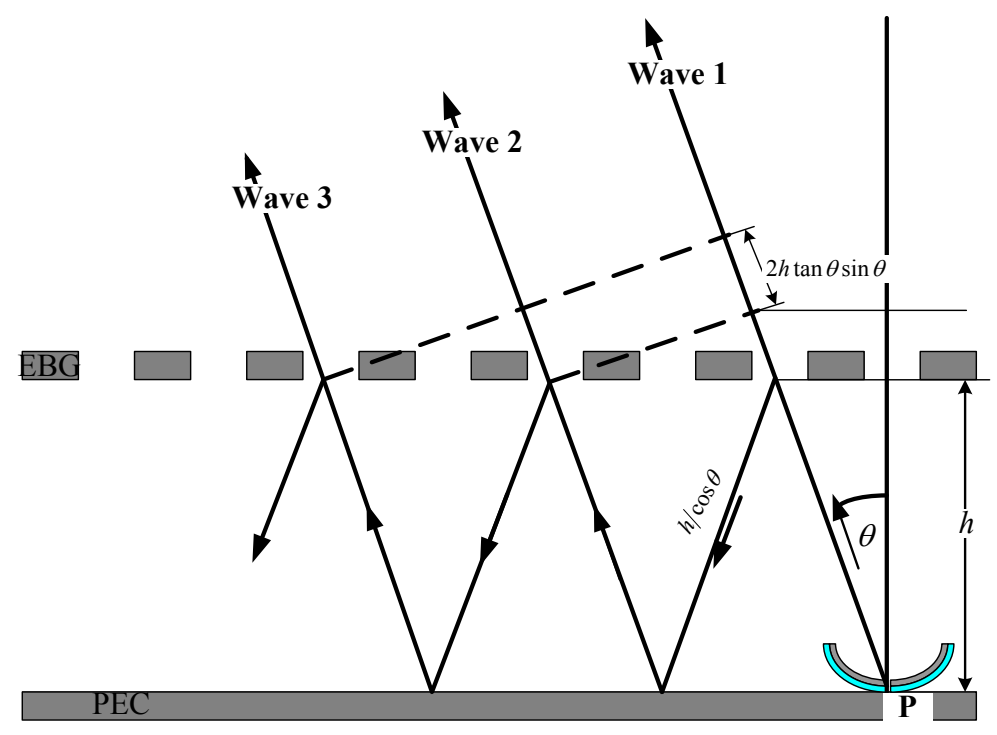

Figure 2. The reflection wave between PEC and EBG.

Assume that the transmission is lossless, the amplitude of the direct wave 1 is $\sqrt{1-\gamma^{2}}$, the amplitude of the once-reflected wave 2 is $\gamma \sqrt{1-\gamma^{2}}$, in the same way the amplitude of the twice-reflected wave 3 is $\gamma^{2} \sqrt{1-\gamma^{2}}$, etc. Therefore, summation of electric field is examined from

$$
\bar{E}=\sum_{n=0}^{\infty} f(\theta) E_{0} \gamma^{n} \sqrt{1-\gamma^{2}} \mathrm{e}^{j \Delta \varphi_{n}},
$$

where, $\Delta \varphi$ is the phase variations. When the phase difference between wave 2 and wave 1 is

$$
\Delta \varphi_{1}=\frac{2 \pi}{\lambda} 2 h \tan \theta \sin \theta-\frac{2 \pi}{\lambda} \frac{2 h}{\cos \theta}-\varphi_{\mathrm{PEC}}+\varphi_{\mathrm{EBG}},
$$

the phase difference between wave 3 and wave 1 is

$$
\Delta \varphi_{2}=\frac{2 \pi}{\lambda} 4 h \tan \theta \sin \theta-\frac{2 \pi}{\lambda} \frac{4 h}{\cos \theta}-2 \varphi_{\mathrm{PEC}}+2 \varphi_{\mathrm{EBG}} .
$$

If the direct wave has $n$ number, so the phase difference is

$$
\Delta \varphi_{n}=n\left[-\frac{4 \pi}{\lambda} \cos \theta-\varphi_{\mathrm{PEC}}+\varphi_{\mathrm{EBG}}\right] .
$$

When $\gamma<1$, we obtain

$$
\sum_{n=0}^{\infty}\left(\gamma \mathrm{e}^{j \Delta \varphi}\right)^{n}=\frac{1}{1+\gamma \mathrm{e}^{j \Delta \varphi}} .
$$

Therefore, we can represent the electric field as follows:

$$
|E|=\left|E_{0}\right| f(\theta) \sqrt{\frac{1-\gamma^{2}}{1+\gamma^{2}-2 \gamma \cos \Delta \varphi}},
$$

and the power pattern is

$$
\bar{S}=\frac{1-\gamma^{2}}{1+\gamma^{2}-2 \gamma \cos \left(\varphi_{\mathrm{EBG}}-\varphi_{\mathrm{PEC}}-\frac{4 \pi}{\lambda} h \cos \theta\right)} f^{2}(\theta) .
$$

However, the amplitude $(\gamma)$ and phase $\left(\varphi_{\mathrm{EBG}}\right)$ of the EBG reflection coefficient are a function of the angle 
$\theta$. Maximum power in the normal direction is obtained, when

$$
\varphi_{\mathrm{EBG}}-\varphi_{\mathrm{PEC}}-\frac{4 \pi}{\lambda} h=0 .
$$

So, the distance between PEC reflector plane and EBG is

$$
h \cong\left(\frac{c}{2 f}\right)\left(\frac{\varphi_{\mathrm{EBG}}-\varphi_{\mathrm{PEC}}}{2 \pi}\right)+N \frac{\lambda}{2},
$$

where, $N=0,1,2,3, \cdots$.

\section{Results and Discussion}

In a previous article, an antenna design of high directive gain using a curved strip dipole on electromagnetic band gap (EBG) was studied [10]. The resonant EBG technology has been used to be a reflector for directive gain increment by utilizing the good performance of the mushroom-like EBG structure, which is capable of providing a constructive image current within a certain frequency band. Therefore, when wide beam curved strip dipole is appropriated located horizontally on a resonant EBG reflector, high directive gain can be obtained of $7.6 \mathrm{dBi}$ for RFID reader. The purpose of this paper is the antenna for mobile base station design, so the antenna performance is developed to perform the resonator antenna by using EBG structure as the superstrate. In this design, the EBG structure can partially reflect wave of a primary radiator. This section proposes the design and analysis of the proposed antenna.

\subsection{The Feeding Antenna}

In Figure 3, the exciting source of the proposed antenna is an upturned curved strip dipole antenna, which is placed on polyvinyl chloride (PVC). So, the radius of a curved strip dipole is specified at 18, 20, 24 and $34 \mathrm{~mm}$. At the radius of $34 \mathrm{~mm}$, it can be obtained the good matching with $50 \mathrm{ohms}$ transmission line. It has the maximum gain of $2 \mathrm{dBi}$ and $S_{11}$ is $-14 \mathrm{~dB}$ at the resonant frequency of $2.1 \mathrm{GHz}$. The pattern of the curved strip dipole in E-plane provides the half power beamwidth (HPBW) around $81.5^{\circ}$ because the dipole is bended to be a half annular and the omni-direction is displayed in H-plane.

\subsection{Circular Polarized Resonator Antenna}

The square circular polarized resonator antenna is excited by a curved strip dipole, oriented along $\phi=45^{\circ}$ direction and placed over the U-shaped reflector plane with the height of $0.2 \lambda$ [15]. Besides, the superstrate consisting of the three EBG layers in polar $\mathrm{V}$, in polar $\mathrm{H}$, and polarizer, is necessary for the two transmitted components to process the equally amplitude and $90^{\circ}$ phase difference. The reflection and transmission phase of the versus frequency are shown in Figure 4(a) to estimate the gap between the reflector and EBG layer as the cavity height $(h)$. The cavity height depends on the frequency which can be obtained through following relations Equation (9) as plotted in Figure 4(b). The parameters $h_{1}$ and $h_{2}$ are $67 \mathrm{~mm}$ and $60 \mathrm{~mm}$, respectively, so, the dual polarized resonator antenna could be produced because of the E-field in horizontal and vertical polarization are equally amplitude.

Furthermore, this paper studies the circularly polarized antenna, so, two perpendicular electromagnetic plane waves not only are equal amplitude but also should have the $90^{\circ}$ phase difference. Therefore, 1-D metallic EBG is designed for the third layer of superstrate, which is called polarizer. The transmission phase of polarizer $\varphi_{S_{21} \text { polarizer }}$ is $-6.24^{\circ}$ as denoted in Figure $4(\mathrm{~b})$. The requirement of phase difference is $90^{\circ}$, where it is given by

$$
k_{0} d_{2}+\varphi_{s_{21} \mathrm{EBGpolarH}}+\varphi_{s_{21} \mathrm{EBGpolarV}}+\varphi_{s_{21} \text { polarizer }}=90^{\circ},
$$

In Equation (10), if the superstrate is separated to find $\varphi_{s_{21}}$, therefore, $d_{2}$ is $23.15 \mathrm{~mm}$. In Figure 5(a), when three layers of EBG structure are used for superstrate of a feeding antenna, the circularly polarized resonator antenna is achieved when the mutual coupling between EBG layers is considered. Figure 5(b) illustrates an axial ratio of square resonator antenna $\left(t_{1}=t_{2}=950 \mathrm{~mm}\right)$, which $d_{2}$ is varied. It seems that an axial ratio close to $0 \mathrm{~dB}$ at the resonant frequency bandwith $d_{2}$ of $30 \mathrm{~mm}$. The maximum gain of $20 \mathrm{~dB}$ could be obtained and the HPBW in vertical and horizontal plane are $18^{\circ}$ and $18.1^{\circ}$, respectively, as shown in Figure 5(c). 


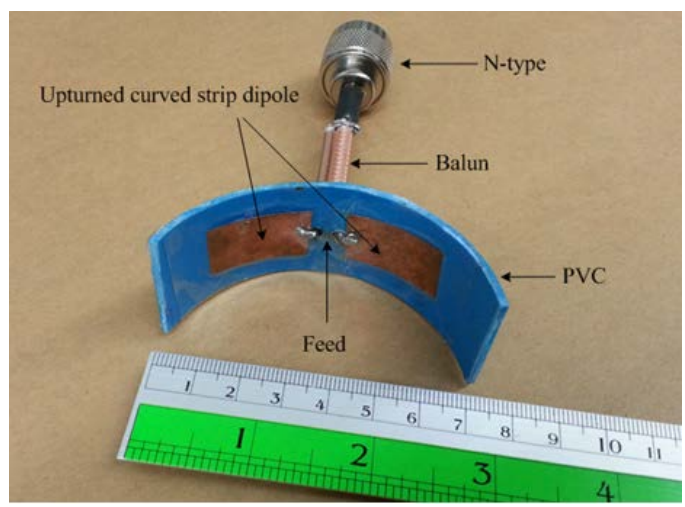

(a)

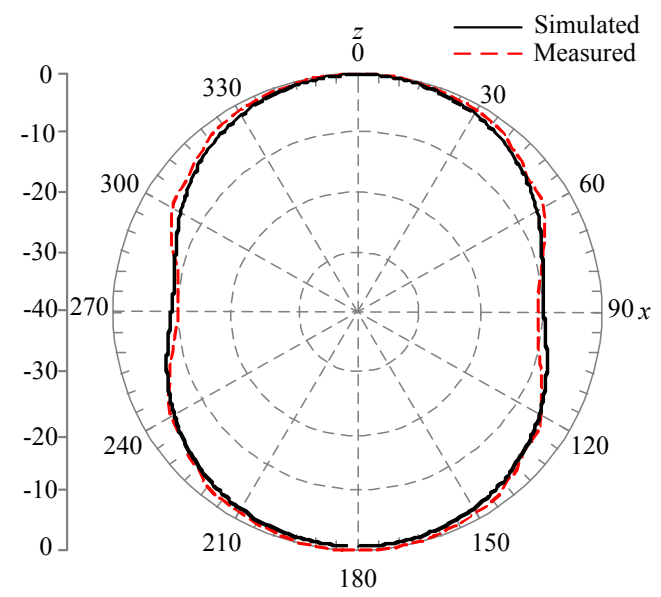

(c)

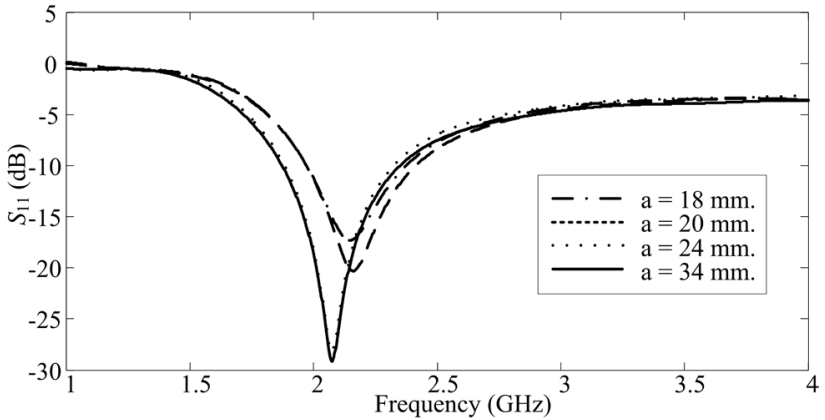

(b)

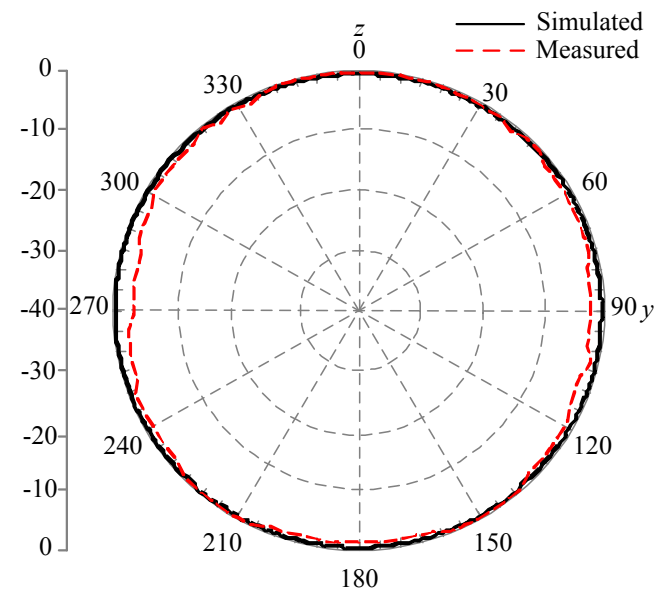

(d)

Figure 3. The results of upturn curved strip dipole antenna: (a) photographs; (b) $S_{11}$; (c) E-plane; (d) H-plane.

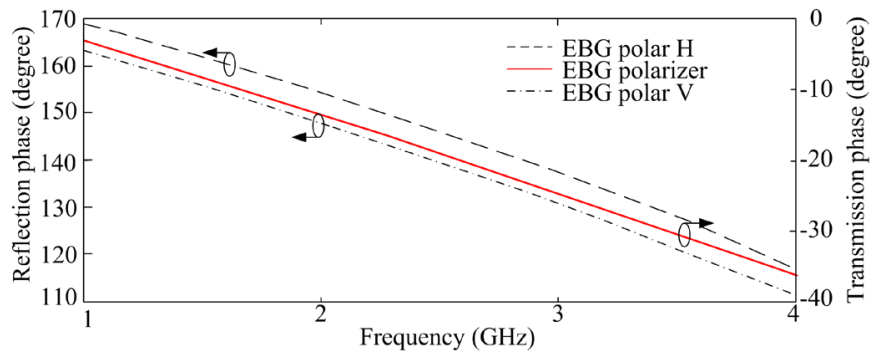

(a)

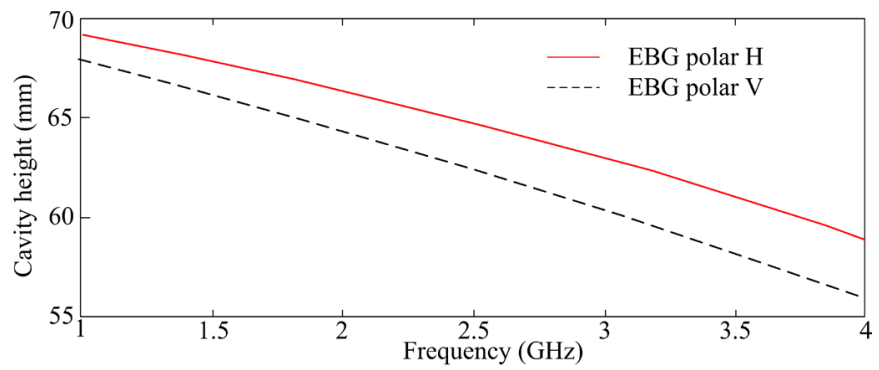

(b)

Figure 4. Simulated results of metallic rod EBG: (a) reflection and transmission phase; (b) calculated cavity height. 




(a)



(c)

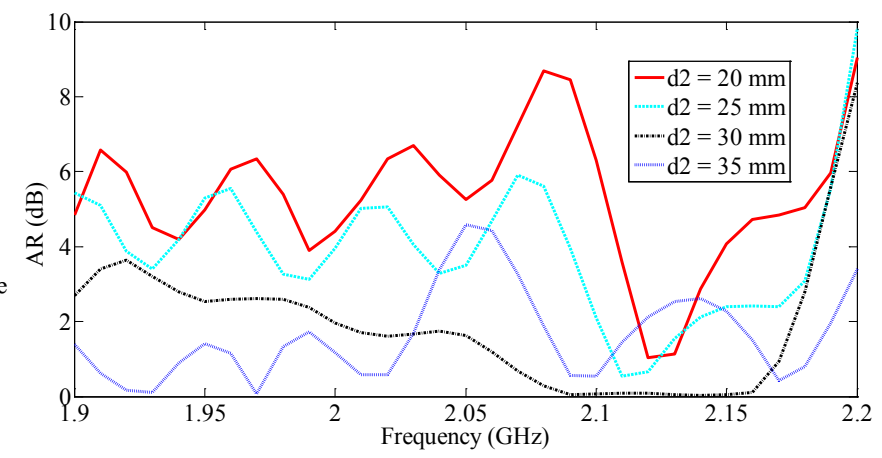

(b)

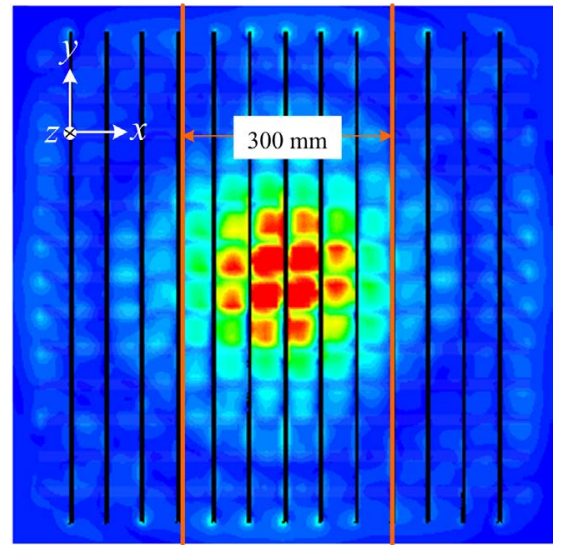

(d)

Figure 5. Simulated results of circularly polarized resonator antenna: (a) structure; (b) AR; (c) normalized radiation patterns; (d) near-field distribution on square reflector plane.

Even if the polarization was achieved in this section, an interesting sectoral $60^{\circ}$ pattern in horizontal plane is the last target of this paper.

\subsection{Sectoral Resonator Antenna with Circular Polarization}

Consideration of Figure 5(d) and Figure 6(a) concludes that if the resonator antenna size $\left(t_{1}\right)$ is reduced to $300 \mathrm{~mm}$ because the near-field level is faded away at the width, the HPBW in horizontal plane of the resonator antenna is wider. While the pattern in horizontal plane is improved, the directive gain of resonator antenna is certainly down. To solve a problem in $x z$ plane, the U-shaped reflector is installed as shown in Figure 2.

To verify the performance of the antenna, an antenna prototype has been fabricated on aluminium as shown in Figure 6(b). The evaluation of the key antenna parameters such as radiation pattern, polarization, and gain were measured by using Network Analyzer. After that the proposed antenna is rotated and the measured radiation pattern of the antenna is plotted together with the simulated pattern as shown in Figure 6(c) and Figure 6(d), respectively. This plot shows agreements between the measured and simulated results both in horizontal and vertical plane patterns. Furthermore, the measured realized gain and AR are continued measure to confirm the antenna performance. Figure 6(e) illustrates an axial ratio close to $0 \mathrm{~dB}$ at the resonant frequency band, covering the uplink and downlink band of $3 \mathrm{G}$ base station. Figure 6(f) shows the measured gain of the proposed antenna. It can be seen that the gain of the antenna is around $15.11 \mathrm{dBi}$ at working range of frequency. The similar radiation and gain enhance the efficiency of mobile communication systems. In addition, some specifications from experiment are compared with the ones from simulation as shown in Table 2. As we can see, the experiment results have a good agreement with the ones from simulation results.

\section{Conclusion}

The design of a circularly polarized resonator antenna using a curved strip dipole with electromagnetic band gap 


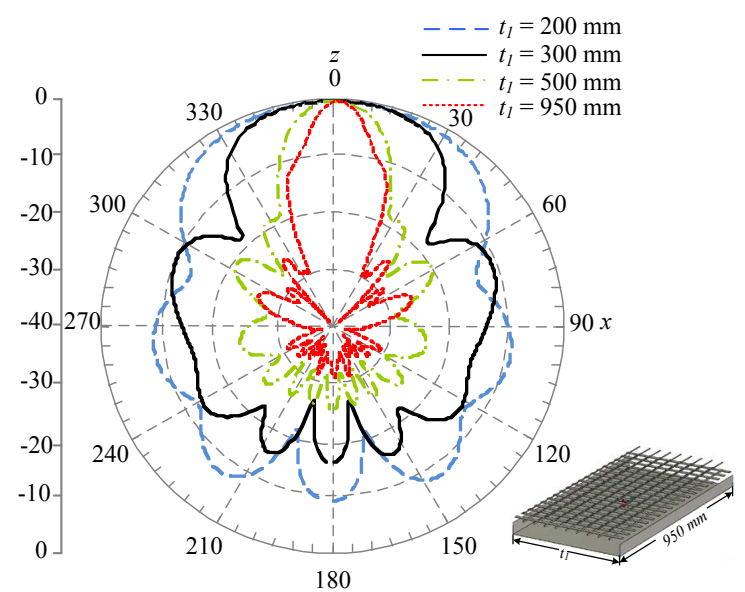

(a)

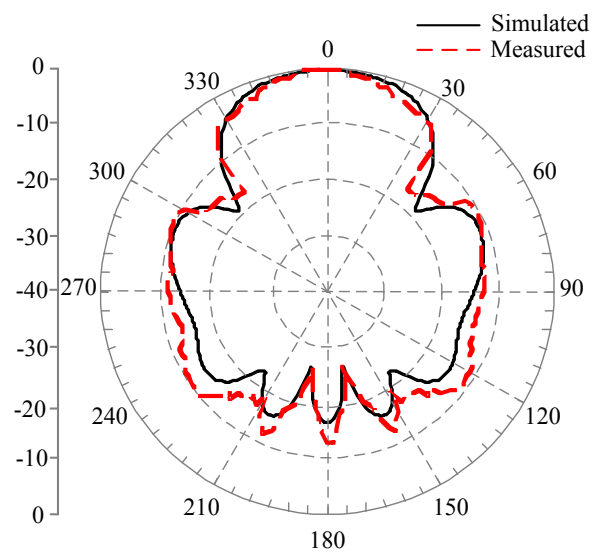

(c)

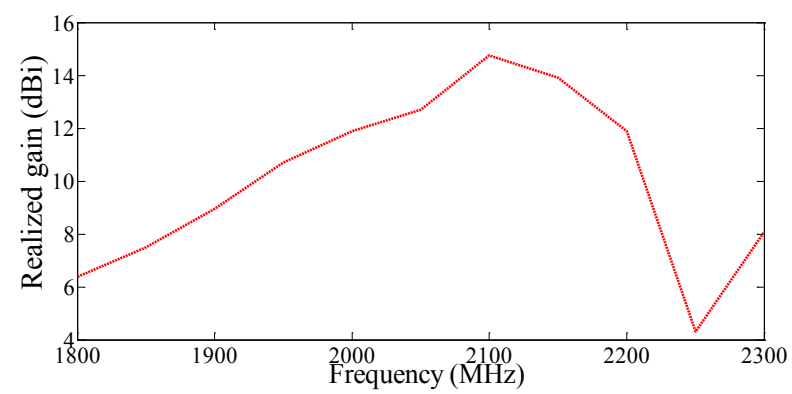

(e)



(b)

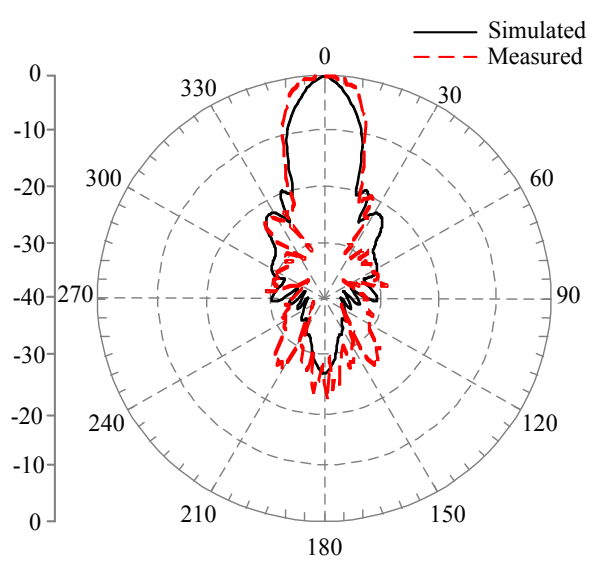

(d)

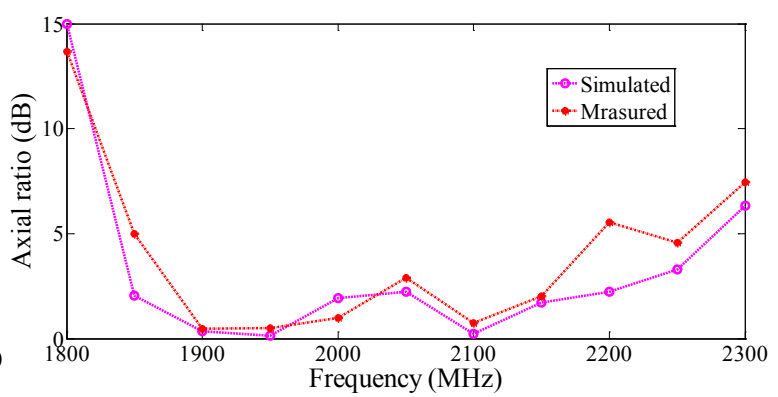

(f)

Figure 6. The results of proposed antenna: (a) horizontal plane when $t_{1}$ is varied; (b) photographs; (c) horizontal plane; (d) vertical plane; (e) realized gain; (f) AR.

material for mobile communications was presented. The antenna was analyzed by using the CST software. From the simulation results, we can conclude that a $45^{\circ}$ oriented curved strip dipole is fed between the mutilayers EBG and the reflector plane to produce the circularly polarization. Furthermore, the sectoral radiation pattern (HPBW around $60^{\circ}$ ) can be obtained by reducing the size of the antenna in $x z$ plane. The antenna prototype was fabricated on aluminium to verify the antenna performance. The measured maximum gain is $15.11 \mathrm{~dB}$. Good agreement between simulated and measured results is obtained.

\section{Acknowledgements}

The authors gratefully acknowledge financial support for this research project from the Telecommunications 
Table 2. Specification of the proposed antenna.

\begin{tabular}{ccccc}
\hline Parameters & \multicolumn{3}{c}{ Simulation } & Measurement \\
\hline Antenna type & Curved strip dipole & Square resonator antenna & Proposed antenna & Proposed antenna \\
Material & Copper plate and PVC & Aluminium & Aluminium & Aluminium \\
Frequency band (MHz) & $2100(1590-2059)$ & $2100(1999-2180)$ & $2100(1850-2230)$ & $2100(1870-2170)$ \\
Polarization & linear & circular & circular & circular \\
HPBW (degree) & E-plane: 81.5 & H: $18.1 \mathrm{~V}: 18$ & H: $60.1 \mathrm{~V}: 12.3$ & H: $60 \mathrm{~V}: 14.4$ \\
Gain (dBi) & 1.63 & 20.11 & 15.53 & 15.11 \\
Antenna size $(\mathrm{mm})$ & $66.75 \times 28$ & $950 \times 950 \times 98$ & $950 \times 300 \times 98$ & $950 \times 300 \times 98$ \\
Antenna weight $(\mathrm{kg})$ & - & - & - & 3.3 \\
\hline
\end{tabular}

Research and Industrial and Development Institute (TRIDI), National Telecommunications Commission (NTC) Fund, Thailand. Moreover, this work was supported by Suranaree University of Technology (SUT) and by the Office of the Higher Education under NRU project of Thailand.

\section{References}

[1] Beckmam, C. and Lindmark, B. (2007) The Evolution of Base Station Antennas for Mobile Communications. International Conference on Electromagnetics in Advanced Applications, Torino, 17-21 September 2007, 85-92.

[2] Abas, A., Asrokin, A., Basri, R.H. and Jamlus, N. (2010) Dual-Polarized Dipole Array Antenna for CDMA 450 Base Station Application. IEEE Asia-Pacific Conference on Applied Electromagnetics (APACE), Port Dickson, 9-11 November 2010, 1-4. http://dx.doi.org/10.1109/APACE.2010.5720102

[3] Azad, M.Z. and Ali, M. (2008) Novel Wideband Directional Dipole Antenna on a Mushroom Like EBG Structure. IEEE Transactions on Antennas and Propagation, 56, 1242-1250. http://dx.doi.org/10.1109/TAP.2008.922673

[4] Zhijun, Z., Iskander, M.F., Langer, J.C. and Mathews, J. (2004) Wideband Dipole Antenna for WLAN. IEEE Antennas and Propagation Society International Symposium, Monterey, 20-25 June 2004, 1963-1966.

[5] Elkamchouchi, H. and Abu Nasr, M. (2004) The S-Shaped Dipole Antenna. 4th International Conference on Microwave and Millimeter Wave Technology Proceeding, Beijing, 18-21 August 2004, 19-22.

[6] Khishnan, L.W. and Leong, M.S. (2005) A V-Shaped Structure for Improving the Directional Properties of the Loop Antenna. IEEE Transactions on Antennas and Propagation, 53, 2114-2117. http://dx.doi.org/10.1109/TAP.2005.848519

[7] Cheng, D.K. (1982) Shaped Wire Antenna with Maximum Directivity. IEEE Transactions on Antennas and Propagation, 18, 816-818.

[8] Wang, J.H., Jen, L. and Jian, S.S. (1997) Optimization of the Dipole Shapes for Maximum Peak Values of the Radiating Pulse. IEEE Antennas and Propagation Society International Symposium, Montreal, Quebec, 13-18 July 1997, 526-529.

[9] Yang, F. and Rahmat-Samii, Y. (2008) Electromagnetic Band Gap Structures in Antenna Engineering. Cambridge University Press, Cambridge. http://dx.doi.org/10.1017/CBO9780511754531

[10] Fhafhiem, N., Krachodnok, P. and Wongsan, R. (2010) Curved Strip Dipole Antenna on EBG Reflector Plane for RFID Applications. WSEAS Transactions on Communications, 9, 374-383.

[11] Pimpol, S. and Wongsan, R. (2014) Band-Notched Printed Dipole Antenna with EBG Reflector. International Conference on Electrical Engineering/Electronics, Computer, Telecommunications and Information Technology, Nakhon Ratchasima, 14-17 May 2014, 1-5.

[12] Abkenar, M.R. and Rezaei, P. (2011) Design of a Novel EBG Structure and Its Application for Improving Performance of a Low Profile Antenna. Iranian Conference on Electrical Engineering (ICEE), Tehran, 17-19 May 2011, 1-5.

[13] Rodes, E., Diblanc, M., Arnaud, E., Monediere, T. and Jecko, B. (2007) Dual-Band EBG Resonator Antenna Using a Single-Layer FSS. IEEE Antennas and Wireless Propagation Letters, 6, 368-371. http://dx.doi.org/10.1109/LAWP.2007.902808

[14] Hajj, M., Rodes, E. and Monediere, T. (2009) Dual-Band EBG Sectoral Antenna Using a Single-Layer FSS for UMTS Application. IEEE Antennas and Wireless Propagation Letters, 8, 161-164. 
http://dx.doi.org/10.1109/LAWP.2009.2012914

[15] Fhafhiem, N., Krachodnok, P. and Wongsan, R. (2013) The Effective Directivity of Resonator Antenna Using Curved Strip Dipole. Asia-Pacific Microwave Conference Proceedings (APMC), Seoul, 5-8 November 2013, 1154-1156.

[16] Frezza, F., Pajewski, L., Piuzzi, E., Ponti, C. and Schettini, G. (2012) Advances in EBG-Resonator Antenna Research. International Symposium on Antennas and Propagation, Nagoys, 29 October-2 November 2012, 1301-1304.

[17] Fhafhiem, N., Krachodnok, P. and Wongsan, R. (2013) Gain Improvement of Curved Strip Dipole Using EBG Resonator. Progress in Electromagnetics Research Symposium Proceedings (PIERS), Kuala Lumpur, 27-30 March 2013, 1631-1634.

[18] Hashmi, R.M., Zeb, B.A. and Esselle, K.P. (2014) Wideband High-Gain EBG Resonator Antennas with Small Footprints and All-Dielectric Superstructures. IEEE Transactions on Antennas and Propagation, 62, 2970-2977. http://dx.doi.org/10.1109/TAP.2014.2314534

[19] Yang, F. and Rahmat-Samii, Y. (2005) A Low Profile Single Dipole Antenna Radiating Circularly Polarized Waves. IEEE Transactions on Antennas and Propagation, 53, 3083-3086. http://dx.doi.org/10.1109/TAP.2005.854536

[20] Hsu, C.-W., Lin, S.-K. and Lin, Y.-C. (2012) Dual-Frequency Dual-Sense Circular Polarization on Asymmetric Crossed-Dipole Antenna. IEEE Antennas and Propagation Society International Symposium, Chicago, 8-14 July 2012, 1-2.

[21] Hajj, M., Monediere, T., Jecko, B. and Chantalat, R. (2010) Gain Improvement of Curved Strip Dipole Using EBG Resonator. International Symposium on Antenna Technology and Applied Electromagnetics \& the American Electromagnetics Conference, Ottawa, 5-8 July 2010, 1-4.

[22] Toubet, M.S., Hajj, M., Chantalat, R., Jecko, B. and Ahmad, A.E. (2011) Generation of Circular Polarization with Low-Profile EBG Antenna. Proceedings of the 5th European Conference on Antennas and Propagation (EUCAP), Rome, 11-15 April 2011, 1177-1180.

[23] Trentini, G.V. (1956) Partially Reflecting Sheet Array. IRE Transactions on Antennas and Propagation, 4, 666-671. http://dx.doi.org/10.1109/TAP.1956.1144455 
Scientific Research Publishing (SCIRP) is one of the largest Open Access journal publishers. It is currently publishing more than 200 open access, online, peer-reviewed journals covering a wide range of academic disciplines. SCIRP serves the worldwide academic communities and contributes to the progress and application of science with its publication.

Other selected journals from SCIRP are listed as below. Submit your manuscript to us via either submit@scirp.org or Online Submission Portal.
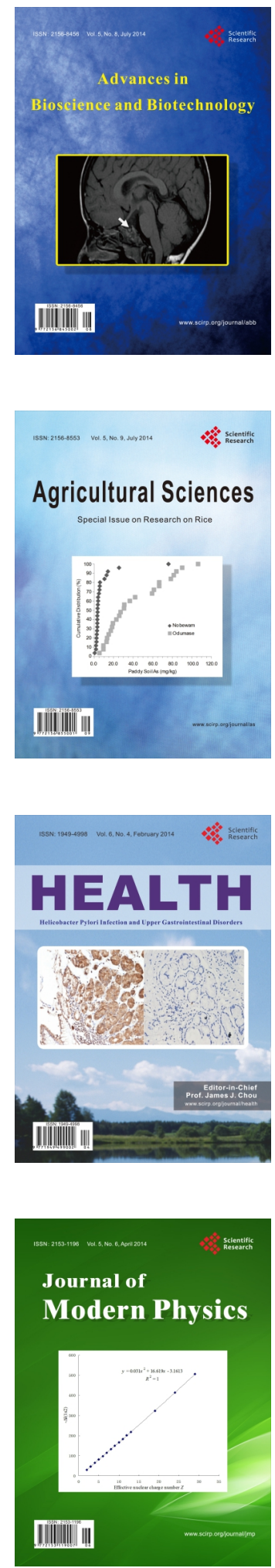
